\title{
Vergleichende Bewertung der Wirkung und klinischen Wirksamkeit von terbinafinhaltigen und bifonazolhaltigen Topika bei der Behandlung der Fußmykose
}

\author{
H. C. Korting ${ }^{1}$ \\ Jutta Kresimon ${ }^{2}$ \\ R. Rychlik ${ }^{2}$
}

\author{
Comparative Evaluation of the Activity and Clinical Effectiveness of Terbinafine \\ and Bifonazole Preparations in the Treatment of Pedal Mycosis
}

\section{Zusammenfassung}

Hintergrund und Fragestellung: Ziel dieser Übersichtsarbeit ist die Darstellung von Wirkmodus, Erregerspektrum sowie mykologischer und klinischer Wirksamkeit von Terbinafin und Bifonazol bei der lokalen Behandlung der Fußmykose. Hierzu werden die Ergebnisse von In-vitro-Untersuchungen und In-vivo-Studien miteinander verglichen und bewertet. Methodik: Die internationale Literaturrecherche erfolgte unter Einbeziehung der Suchbegriffe nach Applikationsart, Erkrankung, Erregerspektrum, Wirkmodus, Wirkstoffen sowie In-vitro-Untersuchungen und In-vivo-Studien. Bei der Analyse der klinischen Studien wurden ausschließlich randomisierte doppelblinde kontrollierte Studien des Evidenzgrades I ausgewählt. Die Diagnose musste klinisch, im Nativpräparat und kulturell gesichert sein. Ergebnisse: In den In-vitro-Untersuchungen zeichneten sich beide Wirkstoffe durch günstige pharmakologische Eigenschaften aus. Terbinafin zeigte jedoch eine deutlich höhere intrinsische Aktivität gegen Dermatophyten, sowie einen primär fungiziden Wirkmodus. Diese überlegenen In-vitro-Eigenschaften fanden auch in klinischen Untersuchungen ihre Entsprechung. Der In-vivoVergleich erfolgte anhand von 12 plazebokontrollierten Studien und zwei direkten Vergleichsstudien. Beide Wirkstoffe zeigten in geeigneter Zubereitung eine unzweifelhafte Überlegenheit gegenüber Plazebo. Die ermittelten Heilungsraten der Vergleichsstudien sind bezüglich Terbinafin-Präparaten zumindest gleichwertig, zeigen jedoch teilweise eine unzweifelhafte Überlegenheit. Dabei waren Terbinafin-Präparationen überlegen durch

\section{Abstract}

Background and Rationale: This review focuses on the in vitro activity and clinical effectiveness of terbinafine and bifonazole in the local treatment of tinea pedis. On the basis of in vitro investigations and in vivo studies a critical comparison is accomplished. Method: The international literature search was based on the search criteria of the description of application, the indication, the activity spectrum, the mode of action of the active substances based on both in vitro investigations and in vivo studies. With the analysis of the clinical trials only randomized double-blind controlled studies of the evidence level of I were selected. The diagnosis had to be confirmed both on clinical and mycological grounds. Results: In vitro investigations revealed with both active substances favourable pharmacologic properties. Terbinafine, however, offered a much higher intrinsic activity against dermatophytes, as well as primary fungicidal activity. These superior in vitro characteristics were reflected in clinical investigations. In vivo comparison took place on the basis of 12 placebo-controlled studies and two direct comparative studies. Both active agents were found superior as compared to placebo. The results of the comparative studies are at least not inferior with respect to terbinafine, while partly an unquestionable superiority of terbinafine was shown. Terbinafine proved superior in comparison to bifonazole by higher effectiveness rates, more rapid onset of clinical improvement after one week of treatment and lower relapse rates. Conclusion: The primarily fungicidal mode of action of terbinafine as well as the immediate penetration into the stratum

Institutsangaben

${ }^{1}$ Klinik und Poliklinik für Dermatologie und Allergologie (Direktor: Prof. Dr. Dr. h.c. G. Plewig) der Ludwig-Maximilians-Universität, München

${ }^{2}$ Institut für Empirische Gesundheitsökonomie (Leiter: Prof. Dr. Dr. med. R. Rychlik), Burscheid

Korrespondenzadresse

Prof. Dr. Hans C. Korting · Klinikum der Universität München · Klinik und Poliklinik für Dermatologie und Allergologie·Frauenlobstraße 9-11·80337 München·E-mail: H.C.Korting@lrz.uni-muenchen.de

Bibliografie

Akt Dermatol 2004; 30: 210-217 @ Georg Thieme Verlag KG Stuttgart · New York · DOI 10.1055/s-2004-814541

ISSN 0340-2541 
schnelleren Wirkungseintritt, hohe Wirksamkeitsraten nach nur 1-wöchiger Behandlung bei Tinea pedis vom interdigitalen Typ und geringere Rezidivraten. Folgerungen: Der primär fungizide Wirkmodus von Terbinafin und die umgehende Penetration ins Stratum corneum mit hohen Wirkstoffkonzentrationen im Gewebe, auch nach Beendigung der Anwendung, vermögen die im Vergleich zu Bifonazol schnellere mykologische und klinische Heilung, eine kürzere Therapiedauer sowie eine geringere Rezidivrate zu erklären. corneum and the presence of high levels in the tissue also after completion of application might explain the shorter time to healing in comparison with bifonazole. The same applies to shorter therapy duration as well as lower relapse rates.

\section{Einleitung}

An eine optimale topische antimykotische Therapie für eine Mykose der freien Haut des Fußes können verschiedene Bewertungskriterien angelegt werden:

- hohe In-vitro-Aktivität gegen die krankheitsauslösenden Erreger,

- gute und schnelle Penetration in die Haut,

- ausreichend lange Hautverweildauer, um eine einmal tägliche Anwendung zu ermöglichen und so die Patientencompliance zu fördern,

- geringe Wirkstoffaufnahme in den Blutkreislauf,

- effektive Heilungsraten,

- kurze Behandlungsdauer,

- Ausbleiben bzw. Minimierung von Hautirritationen oder anderen Nebenwirkungen,

- geringe Rezidiv- bzw. Reinfektionsraten.

Unter diesen Gesichtspunkten sollen die beiden topischen Antimykotika Bifonazol und Terbinafin in dieser Übersichtsarbeit dargestellt und bewertet werden, da ihnen derzeit bei der Behandlung der Fußmykose eine große Bedeutung zukommt - sowohl im Bereich der Verordnung, wie auch in der Selbstmedikation.

\section{Methodik}

\section{Informationsquellen}

Die Recherche der Literatur erfolgte in folgenden Datenbanken: Biosis (BA70) und (BA00), Cochrane Central Register of Controlled Trials (CCTR93), Embase (EM74), Embase Alert (EA08), Medikat (MK77), Medline (ME60), Medline Alert (ME0A), SciSearch (IS74) und (IS00), verfügbar im Deutschen Institut für Medizinische Dokumentation und Information (DIMDI).

\section{Suchstrategie}

Die internationale Literatursuche erfolgte für die Zeitspanne von 1960 bis 2003 unter Einbeziehung der Suchbegriffe zur Erfassung von Applikationsart, Erkrankung, Erregerspektrum, Wirkmodus, Wirkstoffe bezogen auf In-vitro-Untersuchungen und Invivo-Studien. Die gefundenen klinischen Studien wurden bezüglich ihrer methodologischen Qualität Evidenzgraden zugeteilt und Studien mit Evidenzgrad I selektiert [1].

Bei der Selektion der Studien wurden somit ausschließlich randomisierte, doppelblinde kontrollierte klinische Studien in die Analyse einbezogen. Hierbei musste die klinische Diagnose so- wohl durch den Nachweis von Pilzelementen im Nativpräparat als auch durch den kulturellen Pilznachweis mit Erregeridentifikation gesichert sein. Als weitere Voraussetzung galt die Vergleichbarkeit der Patientenkollektive in den Vergleichskohorten. Die mykologische Heilung lag vor, wenn das Nativpräparat und die Kultur negativ waren. Die klinische Heilung bedingte ein Abklingen der klinischen Symptome. Die Gesamtheilung wurde als Vorliegen von mykologischer Heilung und vollständigem bzw. fast vollständigem Verschwinden der Symptome definiert.

\section{Terminologie und Erreger der Fußmykose}

Der Begriff Tinea pedis wird in der medizinischen Literatur synonym für eine Dermatophyteninfektion der freien Haut des Fußes verwendet und beschreibt basierend auf Erregerspektrum und Manifestationsort die häufigste Pilzerkrankung des Fußes [2]. Klinisch werden bei der Tinea pedis drei Erscheinungsformen unterschieden, die interdigitale oder mazerative als die bei weitem häufigste Form, die plantare oder squamös-hyperkeratotische Form, die bei Befall der Fußkanten als Mokassintyp bezeichnet wird, und die vesikulös-dyshidrotische Form [3].

Die unzweifelhaft wichtigsten ursächlichen Erreger der Fußmykosen sind mit ca. 98\% Dermatophyten (Fadenpilze) [4 - 7]. Hefeund Schimmelpilze spielen als Erreger der Fußmykose eine wesentlich geringere Rolle. Hefepilze sind auf der menschlichen Haut ständig anzutreffen und gehören zur normalen Hautflora. Sie werden als so genannte Opportunisten bezeichnet, weil sie bei einer Schwäche ihres Wirtes zu einer Infektion führen und damit pathogen werden [8].

Der mykologische Nachweis von Schimmelpilzen auf der freien Haut des Fußes ist ebenfalls nicht notwendigerweise ein Hinweis auf die pathogene Bedeutung der Keime. Die meisten Schimmelpilze sind lediglich so genannte Anflugkeime, die u. U. bei Abwehrschwäche des Wirtes pathogen werden können. Klinisch bedeutsame Hauterkrankungen des Fußes in Europa, die durch Schimmelpilze verursacht werden, sind am ehesten Nagelpilzinfektionen [3].

Im Folgenden sollen die beiden topischen Antimykotika Bifonazol und Terbinafin hinsichtlich ihrer Wirkung auf Dermatophyten, der Vollständigkeit halber auch Candida sp., sowie ihrer Wirksamkeit in der Therapie der Tinea pedis interdigitalis und Tinea pedis plantaris dargestellt und bewertet werden. 
Bifonazol, ein halogenfreies Imidazol-Derivat, greift an zwei Stellen in die Ergosterol-Biosynthese ein und führt so zu Aufbauund Funktionsstörungen der Zellmembran. Zum einen kommt es zu einer Hemmung des Cytochroms $\mathrm{P}_{450}$, zum anderen hemmt es - im Gegensatz zu früheren Vertretern der Imidazol-Derivate - direkt die HMG-CoA-Reduktase. Dies ist für den fungiziden Wirkmodus von Bifonazol verantwortlich [9].

Untersuchungen zum antimykotischen Wirkmodus belegen, dass Bifonazol nach einer Einwirkzeit von mindestens sechs Stunden in Konzentrationen $\geq 5 \mu \mathrm{g} / \mathrm{ml}$ fungizid auf T. mentagrophytes und T. rubrum wirkt [10]. Bifonazol hemmt das Wachstum der meisten Dermatophytenspezies bei Konzentrationen unter $2,5 \mu \mathrm{g} / \mathrm{ml}[10,11]$. Gegen Hefen und Schimmelpilze wirkt es fungistatisch (Tab. 1) [12].

Pharmakokinetik: Nach topischer Anwendung dringt Bifonazol aufgrund seiner Lipophilie rasch und in hohen Konzentrationen in die Haut ein und bindet dort mit großer Affinität an bestimmte Strukturen wie das Keratin [13].

Bereits nach einer Expositionszeit von 20 bis 30 Minuten wird die maximale Aufnahme von Bifonazol durch die Pilzzelle erreicht. Es verbleibt dort über 100 bis 120 Stunden und unterdrückt während dieser Zeitspanne die Ergosterol-Biosynthese. Die Hautverweildauer in therapeutisch relevanten Konzentrationen beträgt im Mittel 50 bis 60 Stunden [14].

Studien an gesunden Probanden zeigten eine minimale systemische Absorption [13]. Nach einer einzelnen Anwendung von ${ }^{14} \mathrm{C}$-markierter Bifonazol-Creme bzw. -Lösung wurden nach einer 6-stündigen Kontaktperiode lediglich 0,6-0,8\% der radioaktiven Substanz absorbiert. Bei entzündeter Haut erhöhte sich die Absorptionsrate um das 3- bis 5 fache, blieb jedoch unter $4 \%$.

\section{Terbinafin}

Terbinafin hemmt die Squalenepoxidase und führt darüber zu einem Mangel an Ergosterol, einer essenziellen Zellmembrankomponente, und einer Akkumulation von Squalen in Form von Lipidvesikeln in der Pilzzelle mit Freisetzung lytischer Enzyme und nachfolgendem Zelltod [15]. In vitro wirkt Terbinafin primär fungizid gegen Dermatophyten [16]. Gegen Schimmelpilze und Hefen zeigt sich je nach Spezies eine fungizide oder fungistatische Wirkung.

Pharmakokinetik: Terbinafin diffundiert nach topischer Applikation in die oberen Schichten des Stratum corneum und bindet dort aufgrund seiner lipophilen Natur effektiv an die Keratozyten. Es akkumuliert in Stratum corneum, Nägeln, Haaren, Dermis/Epidermis sowie in Fettgewebe [17]. Hill et al.[18] ermittelten in einer Studie zur Pharmakokinetik von 1 \%iger TerbinafinCreme, dass die maximale Konzentration $\left(C_{\max }\right)$ im Stratum corneum $\left(94,9 \mu \mathrm{g} / \mathrm{m}^{2}\right)$ vier Stunden nach einer einzelnen Applikation erreicht wurde. Bei Verlängerung der Behandlungsdauer auf sieben Tage erhöht sich $C_{\max }$ nur um $15 \%$, die Eliminationshalbwertszeit im Stratum corneum jedoch um das Dreifache.
Tab. 1 Zusammenfassung der In-vitro-Aktivität von Bifonazol und Terbinafin gegen die häufigsten Tinea pedis auslösenden Dermatophytenspezies [nach 10, 11, 16, 21, 22, 23], Hefeund Schimmelpilze [nach 10, 21, 23, 24]

\begin{tabular}{|lcc}
\hline & Bifonazol & Terbinafin \\
\hline Dermatophyten & MHK-Werte (mg/l) & MHK-Werte (mg/l) \\
\hline T. mentagrophytes & 2,5 & $0,001-0,01$ \\
\hline T. rubrum & 0,63 & $0,001-0,01$ \\
\hline E. floccosum & $<0,04$ & $0,001-<0,06$ \\
\hline M. canis & 1,25 & $0,005-0,01$ \\
\hline M. gypseum & $0,03-5$ & 0,03 \\
\hline M. audouinii & 2,5 & 0,03 \\
\hline Hefen & MHK-90\%-Werte (mg/I) & MHK-Werte (mg/I) \\
\hline C. albicans & $0,23-20$ & $0,03->40$ \\
\hline C. tropicalis & $1,87-20$ & $0,11->40$ \\
\hline C. parapsilosis & $1,25-10$ & $0,13-3,13$ \\
\hline C. glabrata & $0,32-20$ & $0,17->40$ \\
\hline C. famata & 10 & 0,15 \\
\hline C. humicola & 20 & 20 \\
\hline C. guilliermondii & $1,87-5$ & $0,03-20$ \\
\hline C. krusei & $0,93-5$ & $0,03->40$ \\
\hline C. intermedia & 10 & 40 \\
\hline Schimmelpilze & MHK-90\%-Werte (mg/l) & MHK-Werte (mg/l) \\
\hline Aspergillus fumigatus & $1-2$ & $0,05-1,56$ \\
\hline Aspergillus ssp. & $1-4$ & - \\
\hline & & \\
\hline & &
\end{tabular}

Die gemessenen Konzentrationen waren um den Faktor 1000 höher als die fungiziden Konzentrationen für T. rubrum und andere bekannte Dermatophyten. Selbst sieben Tage nach Beendigung der Behandlung wurden noch 100-mal höhere und fungizide Konzentrationen im Stratum corneum gemessen.

Auch bei der hyperkeratotischen Form der Tinea pedis konnten Wirkstoffkonzentrationen im Stratum corneum nachgewiesen werden, die um ein Vielfaches höher lagen als die MHK-Werte (minimale Hemmkonzentration) für Dermatophyten [19].

Das Eindringen von Terbinafin in den Blutkreislauf nach topischer Anwendung ist minimal. Nach perkutaner Applikation von $1 \%$ iger Terbinafin-Creme bei Gesunden und Patienten mit Pityriasis versicolor konnten nur bei wenigen Probanden messbare Plasmakonzentrationen gefunden werden [20].

Kritische Bewertung der Ergebnisse der präklinischen Wirkung der beiden Wirkstoffe Bifonazol und Terbinafin

Die verschiedenen In-vitro-Methoden sind heute weitgehend standardisiert und zuverlässig, dennoch sind die Ergebnisse der einzelnen Untersucher nur bedingt miteinander vergleichbar, da die Bestimmung der Hemmwirkung von der Zusammensetzung und dem pH-Wert des Nährmediums sowie von der Größe des Inokulums abhängig ist. Es wurden z.T. unterschiedliche Verdünnungsmethoden und Nährmedien verwendet.

Trotz begrenzter Vergleichbarkeit der einzelnen Untersuchungen lassen sich wesentlich niedrigere minimale Hemmkonzen- 
trationen für Terbinafin im Gegensatz zu Bifonazol bei den bedeutsamen Dermatophytenspezies erkennen.

Eine Steigerung der Applikationsfrequenz von Terbinafin erhöht die maximale Wirkstoffkonzentration im Stratum corneum nicht signifikant, bereits eine einmal tägliche Verabreichung führt zu effektiven Ergebnissen [19]. Auch nach Beendigung der Therapie konnten Terbinafin-Spiegel nachgewiesen werden, die signifikant höher waren als die fungiziden Konzentrationen für alle bekannten Dermatophyten, so dass eine kurzzeitige Behandlung von sieben Tagen greifbar erscheint.

Untersuchungen zur Tiefenlokalisation ließen auch für Bifonazol ein gutes Penetrationsvermögen der Substanz in die Haut erkennen. Noch in den unteren Schichten der Epidermis wurden die in vitro bestimmten MHK-Werte für Dermatophyten um ein Vielfaches überschritten [13]. Die Langzeit-Retention von Bifonazol in der Haut ermöglicht ebenfalls eine einmal tägliche Anwendung, jedoch sollte die Behandlung drei Wochen erfolgen.

Trotz besserer In-vitro-Ergebnisse für Terbinafin können diese nicht ohne weiteres auf eine In-vivo-Situation übertragen werden, sie müssen vielmehr gegebenenfalls in klinischen Studien bestätigt werden.

\section{Klinische Wirksamkeit}

Durch die Einteilung der klinischen Studien in Evidenzgrade konnten 14 Studien des Evidenzgrades I ermittelt werden. In vier Studien wurde Bifonazol mit Plazebo, in acht Studien Terbinafin mit Plazebo und in zwei Studien Bifonazol mit Terbinafin verglichen.

\section{Vergleichsstudien Bifonazol versus Plazebo (Tab. 2)}

Die Wirksamkeit von Bifonazol wurde an insgesamt 182 Patienten mit der Indikation Tinea pedis interdigitalis überprüft. Der Wirkstoff wurde einmal täglich für einen Behandlungszeitraum von vier Wochen als 1 \%ige Creme oder 1 \%ige Lösung angewandt. Der Beobachtungszeitraum betrug sechs Wochen.

Mykologische Heilungsraten:

Bifonazol: 64-91\%

Plazebo: $22-67 \%$

Klinische Heilungsraten:

Bifonazol: 64-95\%

Plazebo: $13-48 \%$

Gesamtheilungsraten:

Bifonazol: $52-70 \%$

Plazebo: $10-29 \%$

Alle Studien zeigten eine signifikante Überlegenheit des Azolpräparates mit Bifonazol gegenüber Plazebo.

Für die Indikation Tinea pedis plantaris existieren keine Arbeiten, die eine topische Behandlung mit Bifonazol entsprechend den festgelegten Kriterien bzw. eine alleinige Therapie mit Bifonazol dokumentieren.

\section{Vergleichsstudien Terbinafin versus Plazebo (Tab. 3)}

703 Patienten mit der Diagnose Tinea pedis interdigitalis und/ oder Tinea pedis plantaris wurden 1- bis 2-mal täglich für eine Woche bis vier Wochen topisch entweder mit einem TerbinafinPräparat (1\%ige Creme, Lösung oder Gel) oder mit Placebo therapiert. Der Beobachtungszeitraum betrug 6 bis 8 Wochen.

Mykologische Heilungsraten:

Terbinafin: $81-100 \%$

Plazebo: $8-45 \%$

Klinische Heilungsraten:

Terbinafin: $66-79 \%$

Plazebo: $4-44 \%$

Tab. 2 Vergleichsstudien Bifonazol versus Plazebo bei Tinea pedis interdigitalis

\begin{tabular}{|c|c|c|c|c|c|c|c|c|c|}
\hline Autor & Indikationsgebiet & Gruppe & $\begin{array}{l}\text { Patienten- } \\
\text { anzahl }\end{array}$ & $\begin{array}{l}\text { Anwendungen } \\
\text { pro Tag }\end{array}$ & $\begin{array}{l}\text { Behandlungs- } \\
\text { dauer } \\
\text { (Wochen) }\end{array}$ & $\begin{array}{l}\text { Beobachtungs- } \\
\text { zeitraum } \\
\text { (Wochen) }\end{array}$ & $\begin{array}{l}\text { Heilungsrate } \\
\text { mykologisch } \\
(\%)\end{array}$ & $\begin{array}{l}\text { klinisch } \\
\text { (\%) }\end{array}$ & $\begin{array}{l}\text { Gesamtheilung } \\
\text { (\%) }\end{array}$ \\
\hline $\begin{array}{l}\text { Bagatell } 1986 \\
\text { [25] }\end{array}$ & $\begin{array}{l}\text { Tinea pedis } \\
\text { interdigitalis }\end{array}$ & $\begin{array}{l}\text { Bifonazol- } \\
\text { Lösung } 1 \% \\
\text { Plazebo }\end{array}$ & $\begin{array}{l}26 \\
21\end{array}$ & qd & 4 & 6 & $\begin{array}{l}73 \\
48\end{array}$ & $\begin{array}{l}81 \dagger \\
48\end{array}$ & $\begin{array}{l}69 \dagger \\
29\end{array}$ \\
\hline $\begin{array}{l}\text { Coffey } 1986 \\
{[26]}\end{array}$ & $\begin{array}{l}\text { Tinea pedis } \\
\text { interdigitalis }\end{array}$ & $\begin{array}{l}\text { Bifonazol- } \\
\text { Creme 1\% } \\
\text { Plazebo }\end{array}$ & $\begin{array}{l}25 \\
18\end{array}$ & $\mathrm{qd}$ & 4 & 6 & $\begin{array}{l}64 \dagger \\
22\end{array}$ & $\begin{array}{l}64 \dagger \\
17\end{array}$ & $\begin{array}{l}52 \dagger \\
17\end{array}$ \\
\hline $\begin{array}{l}\text { Izuno } 1986 \\
\text { [27] }\end{array}$ & $\begin{array}{l}\text { Tinea pedis } \\
\text { interdigitalis }\end{array}$ & $\begin{array}{l}\text { Bifonazol- } \\
\text { Creme 1\% } \\
\text { Plazebo }\end{array}$ & $\begin{array}{l}20 \\
18\end{array}$ & qd & 4 & 6 & $\begin{array}{l}90^{*} \\
67\end{array}$ & $\begin{array}{l}95 \\
44\end{array}$ & $\begin{array}{l}70 \\
28\end{array}$ \\
\hline $\begin{array}{l}\text { Smith et al. } \\
1986 \text { [28] }\end{array}$ & $\begin{array}{l}\text { Tinea pedis } \\
\text { interdigitalis }\end{array}$ & $\begin{array}{l}\text { Bifonazol- } \\
\text { Lösung } 1 \% \\
\text { Plazebo }\end{array}$ & $\begin{array}{l}23 \\
31\end{array}$ & qd & 4 & 6 & $\begin{array}{l}91 \ddagger \\
23\end{array}$ & $\begin{array}{l}70 \ddagger \\
13\end{array}$ & $\begin{array}{l}70 \dagger \\
10\end{array}$ \\
\hline
\end{tabular}

k. A. = keine Angabe, qd = $1 \times$ täglich, bid $=2 \times$ täglich; $\dagger) p \leq 0,05$ vs. Plazebo; $\ddagger$ ) $p \leq 0,001$ vs. Plazebo; ${ }^{*}$ ) nicht signifikant 
Tab. 3 Vergleichsstudien Terbinafin versus Plazebo

\begin{tabular}{|c|c|c|c|c|c|c|c|c|c|}
\hline Autor & Indikationsgebiet & Gruppe & $\begin{array}{l}\text { Patienten- } \\
\text { anzahl }\end{array}$ & $\begin{array}{l}\text { Anwendungen } \\
\text { pro Tag }\end{array}$ & $\begin{array}{l}\text { Behandlungs- } \\
\text { dauer } \\
\text { (Wochen) }\end{array}$ & $\begin{array}{l}\text { Beobachtungs- } \\
\text { zeitraum } \\
\text { (Wochen) }\end{array}$ & $\begin{array}{l}\text { Heilungsrate } \\
\text { mykologisch } \\
\text { (\%) }\end{array}$ & $\begin{array}{l}\text { klinisch } \\
\text { (\%) }\end{array}$ & $\begin{array}{l}\text { Gesamtheilung } \\
\text { (\%) }\end{array}$ \\
\hline $\begin{array}{l}\text { Berman et al. } \\
1992 \text { [29] }\end{array}$ & $\begin{array}{l}\text { Tinea pedis } \\
\text { interdigitalis }\end{array}$ & $\begin{array}{l}\text { Terbinafin- } \\
\text { Creme } 1 \% \\
\text { Plazebo }\end{array}$ & $\begin{array}{l}80 \\
79\end{array}$ & bid & 1 & 6 & $\begin{array}{l}88 \ddagger \\
23\end{array}$ & $\begin{array}{l}79 \ddagger \\
44\end{array}$ & $\begin{array}{l}\text { k. A. } \\
\text { k. A. }\end{array}$ \\
\hline $\begin{array}{l}\text { Hollmen et al. } \\
2002 \text { [30] }\end{array}$ & $\begin{array}{l}\text { Tinea pedis } \\
\text { interdigitalis }\end{array}$ & $\begin{array}{l}\text { Terbinafin- } \\
\text { Gel } 1 \% \\
\text { Plazebo }\end{array}$ & $\begin{array}{l}39 \\
31\end{array}$ & qd & 1 & $>8$ & $\begin{array}{l}82 \ddagger \\
32\end{array}$ & $\begin{array}{l}\text { k.A. } \\
\text { k.A. }\end{array}$ & $\begin{array}{l}64 t \\
26\end{array}$ \\
\hline $\begin{array}{l}\text { Korting et al. } \\
2001[31]\end{array}$ & $\begin{array}{l}\text { Tinea pedis } \\
\text { interdigitalis }\end{array}$ & $\begin{array}{l}\text { Terbinafin- } \\
\text { Creme } 1 \% \\
\text { Plazebo }\end{array}$ & $\begin{array}{l}50 \\
50\end{array}$ & qd & 1 & 8 & $\begin{array}{l}91 \ddagger \\
37\end{array}$ & $\begin{array}{l}\text { k.A. } \\
\text { k.A. }\end{array}$ & $\begin{array}{l}74 \dagger \\
26\end{array}$ \\
\hline $\begin{array}{l}\text { Lebwohl et al. } \\
2001 \text { [32] }\end{array}$ & $\begin{array}{l}\text { Tinea pedis } \\
\text { interdigitalis }\end{array}$ & $\begin{array}{l}\text { Terbinafin- } \\
\text { Lösung } 1 \% \\
\text { Plazebo }\end{array}$ & $\begin{array}{l}58 \\
28\end{array}$ & bid & 1 & 8 & $\begin{array}{l}96 \ddagger \\
36\end{array}$ & $\begin{array}{c}66 \ddagger \\
4\end{array}$ & $\begin{array}{l}\text { k. A. } \\
\text { k. A. }\end{array}$ \\
\hline $\begin{array}{l}\text { Savin } 1990 \\
{[33]}\end{array}$ & $\begin{array}{l}\text { Tinea pedis } \\
\text { interdigitalis und } \\
\text { Mokassintyp }\end{array}$ & $\begin{array}{l}\text { Terbinafin- } \\
\text { Creme } 1 \% \\
\text { Plazebo }\end{array}$ & $\begin{array}{l}13 \\
14\end{array}$ & bid & 4 & 6 & $\begin{array}{r}100 \\
8\end{array}$ & $\begin{array}{l}\text { k. A. } \\
\text { k.A. }\end{array}$ & $\begin{array}{c}89 \ddagger \\
0\end{array}$ \\
\hline $\begin{array}{l}\text { Smith et al. } \\
1990 \text { [34] }\end{array}$ & $\begin{array}{l}\text { Tinea pedis } \\
\text { interdigitalis und } \\
\text { Mokassintyp }\end{array}$ & $\begin{array}{l}\text { Terbinafin- } \\
\text { Creme } 1 \% \\
\text { Plazebo }\end{array}$ & $\begin{array}{l}10 \\
10\end{array}$ & bid & 4 & 6 & $\begin{array}{l}88 \\
10\end{array}$ & $\begin{array}{l}\text { k. A. } \\
\text { k.A. }\end{array}$ & $\begin{array}{c}78 \ddagger \\
0\end{array}$ \\
\hline $\begin{array}{l}\text { Savin et al. } \\
1994 \text { [35] }\end{array}$ & $\begin{array}{l}\text { Tinea pedis } \\
\text { Mokassintyp }\end{array}$ & $\begin{array}{l}\text { Terbinafin- } \\
\text { Creme } 1 \% \\
\text { Plazebo }\end{array}$ & $\begin{array}{l}97 \\
96\end{array}$ & bid & 2 & 8 & $\begin{array}{l}84 \ddagger \\
21\end{array}$ & $\begin{array}{l}79 \ddagger \\
28\end{array}$ & $\begin{array}{l}\text { k. A. } \\
\text { k. A. }\end{array}$ \\
\hline $\begin{array}{l}\text { Evans et al. } \\
1991 \text { [36] }\end{array}$ & Tinea pedis\# & $\begin{array}{l}\text { Terbinafin- } \\
\text { Creme } 1 \% \\
\text { Plazebo }\end{array}$ & $\begin{array}{l}28 \\
20\end{array}$ & qd & 2 & 6 & $\begin{array}{l}81 \dagger \\
45\end{array}$ & $\begin{array}{l}\text { k.A. } \\
\text { k.A. }\end{array}$ & $\begin{array}{l}68 \dagger \\
35\end{array}$ \\
\hline
\end{tabular}

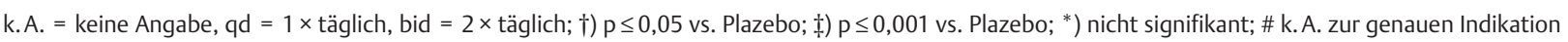

Gesamtheilungsraten:

Terbinafin: $64-89 \%$

Plazebo: $0-35 \%$

In allen Untersuchungen konnte eine signifikante Überlegenheit des Allylaminpräparates mit Terbinafin gegenüber Plazebo festgestellt werden.

\section{Vergleichsstudien Terbinafin versus Bifonazol (Tab. 4)}

Insgesamt wurden 120 Patienten mit Tinea pedis mit Terbinafinbzw. Bifonazol-Creme 1\% behandelt. Der Beobachtungszeitraum lag zwischen 6 und 8 Wochen.

Mykologische Heilungsraten:

Terbinafin: $90-100 \%$

Bifonazol: $79-100 \%$

Klinische Heilungsraten:

Terbinafin: $100 \%$

Bifonazol: $95-100 \%$

Gesamtheilungsraten:

Terbinafin: $78-83 \%$

Bifonazol: $31-63 \%$

Die Gesamtheilungsrate zeigt eine signifikante Überlegenheit von Terbinafin. Ebenfalls konnte ein schnellerer Wirkungseintritt von Terbinafin im Vergleich zu Bifonazol beobachtet werden.

\section{Nebenwirkungen}

In 14 klinischen Studien wurden insgesamt 1005 Patienten mit topischen Wirkstoff- bzw. Plazebopräparaten behandelt. Die dokumentierten Nebenwirkungen waren größtenteils mild und traten in Form von lokalen Hautirritationen, Rötungen, Brennen und Juckreiz auf. In vier plazebokontrollierten Studien und zwei Vergleichsstudien wurde die Verträglichkeit von Bifonazol betrachtet. Hierbei traten bei fünf von 147 Patienten (3,4\%) Nebenwirkungen auf, die jedoch in keinem Fall zu einem vorzeitigen Abbruch der Therapie führten. Die Verträglichkeit von Terbinafin wurde in acht plazebokontrollierten Studien und zwei Vergleichsstudien dokumentiert. Insgesamt wurden 442 Probanden in die Untersuchungen einbezogen, typische Hautirritationen traten in 2,5\% der Fälle auf. Das Auftreten eines erythematösen Ausschlages an Händen und Armen eines Patienten führte zum vorzeitigen Abbruch der Therapie. Bei 2,2\% der plazebobehandelten Patienten (9 von 416) traten ebenfalls lokale Nebenwirkungen auf, die jedoch bei keinem Patienten einen vorzeitigen Therapieabbruch bedingten. Die Verträglichkeit der Antimykotika liegt somit in der gleichen Größenordnung wie die der Plazebopräparate.

\section{Rezidivraten}

Vier Studien betrachteten die plazebokontrollierte Anwendung eines Bifonazol-Präparates, wobei keiner dieser Untersuchungen Angaben zu Rezidivraten entnommen werden konnten. Weiterhin wurde in acht Studien über die plazebokontrollierte Applikation des Wirkstoffes Terbinafin berichtet. Hierbei enthielten drei 
Tab. 4 Vergleichsstudien Terbinafin versus Bifonazol

\begin{tabular}{|c|c|c|c|c|c|c|c|c|c|c|c|c|c|c|c|}
\hline \multirow[t]{2}{*}{ Autor } & \multirow[t]{2}{*}{$\begin{array}{l}\text { Indikations- } \\
\text { gebiet }\end{array}$} & \multirow[t]{2}{*}{ Gruppe } & \multirow[t]{2}{*}{$\begin{array}{l}\text { Patienten- } \\
\text { anzahl }\end{array}$} & \multirow[t]{2}{*}{$\begin{array}{l}\text { Anwendungen } \\
\text { pro Tag }\end{array}$} & \multirow[t]{2}{*}{$\begin{array}{l}\text { Behandlungs- } \\
\text { dauer } \\
\text { (Wochen) }\end{array}$} & \multirow[t]{2}{*}{$\begin{array}{l}\text { Beobachtungs- } \\
\text { zeitraum } \\
\text { (Wochen) }\end{array}$} & \multicolumn{4}{|c|}{$\begin{array}{l}\text { Heilungsrate } \\
\text { mykologisch (\%) }\end{array}$} & \multicolumn{4}{|c|}{ klinisch (\%) } & \multirow[t]{2}{*}{$\begin{array}{l}\text { Gesamt- } \\
\text { heilung (\%) }\end{array}$} \\
\hline & & & & & & & $2 w$ & & $8 w$ & & & & & & \\
\hline \multirow[t]{3}{*}{$\begin{array}{l}\text { Wahid et al. } \\
1997 \text { [37] }\end{array}$} & $\begin{array}{l}\text { Tinea pedis } \\
\text { Mokassintyp }\end{array}$ & $\begin{array}{l}\text { Terbinafin- } \\
\text { Creme 1\% }\end{array}$ & 40 & qd & 2 & 8 & 43 & & 90 & & k. A. & & & & $83 \dagger$ \\
\hline & & $\begin{array}{l}\text { Bifonazol- } \\
\text { Creme 1\% }\end{array}$ & 38 & qd & 2 & 8 & 42 & & 79 & & k. A. & & & & $63 \dagger$ \\
\hline & & & & & & & $1 \mathrm{~W}$ & $2 w$ & $3 w$ & $4 W$ & $1 \mathrm{w}$ & $2 w$ & $3 w$ & $4 w$ & \\
\hline \multirow{2}{*}{$\begin{array}{l}\text { Sanchez } \\
\text { Caruso } \\
1994 \text { [38] }\end{array}$} & $\begin{array}{l}\text { Tinea pedis } \\
\text { interdigitalis }\end{array}$ & $\begin{array}{l}\text { Terbinafin- } \\
\text { Creme } 1 \%\end{array}$ & 27 & qd & 4 & 6 & 4 & $77 \ddagger$ & 96 & 100 & 4 & $73 \ddagger$ & 92 & 100 & $78 \dagger$ \\
\hline & & $\begin{array}{l}\text { Bifonazol- } \\
\text { Creme 1\% }\end{array}$ & 15 & qd & 4 & 6 & 0 & $25 \ddagger$ & 75 & 100 & 0 & $25 \ddagger$ & 69 & 100 & $31 \dagger$ \\
\hline
\end{tabular}

k. A. = keine Angabe, qd = $1 \times$ täglich, bid $=2 \times$ täglich, $W=$ Woche; $\dagger) p \leq 0,05 ;$ ) $p \leq 0,001 ;{ }^{*}$ ) nicht signifikant

Dokumentationen Angaben zu Rezidivraten, in einer Studie trat keine Reinfektion auf und vier Publikationen beinhalteten keine Angaben bezüglich des Auftretens von Rezidiven.

Zwei Studien dokumentierten den direkten Vergleich der beiden Wirkstoffe, wobei einer Untersuchung [37] keine Angaben zu Rezidivraten entnommen werden konnten.

Sowohl Lebwohl et al. [32] als auch Hollmen et al. [30] dokumentierten eine 1-wöchige Behandlung der Tinea pedis mit Terbinafin bei 58 bzw. 39 Patienten. Nach einem Beobachtungszeitraum von acht Wochen wurde eine Reinfektion bei einem (3\%) bzw. vier Patienten (7\%) nachgewiesen. Nach einer 4-wöchigen Behandlung der Tinea pedis mit Terbinafin dokumentierten Smith et al. [34] eine mykologische Rezidivrate von $12 \%$.

Sanchez et al. [38] wiesen bei Studienteilnehmern mit Tinea pedis nach einer 4-wöchigen Behandlung mit Terbinafin (27 Probanden) bzw. Bifonazol (15 Probanden) klinische Rezidivraten von $7,4 \%$ bzw. 6,3\% nach. Eine mykologische Reinfektion konnte für die Terbinafin-Gruppe nicht festgestellt werden, trat jedoch bei $12,5 \%$ der Bifonazol-Patienten ein.

Eine weitere Studie [39], bei der die Teilnehmer bereits durch eine topische Behandlung mit Bifonazol eine mykologische und klinische Heilung erfahren hatten, kann als Langzeit-Follow-up betrachtet werden. Eine Probandengruppe wurde sechs Monate prophylaktisch mit Bifonazol-Puder $1 \%$ behandelt, während die anderen Studienteilnehmer ein Plazebo applizierten. Nach diesen 6 Monaten wurde in der Plazebogruppe ohne Bifonazolprophylaxe eine Rezidivrate von 56\% diagnostiziert, während in der Verumgruppe eine Rezidivrate von $11,5 \%$ auftrat.

Im direkten Vergleich der beiden Antimykotika waren die Rezidivraten in einer Studie miteinander vergleichbar, während die zweite Studie eine deutliche Überlegenheit von Terbinafin (12\% zu 20\%) aufzeigte.
Kritische Bewertung der klinischen Ergebnisse der beiden Wirkstoffe Bifonazol und Terbinafin

Die Literaturrecherche wies 14 randomisierte, doppelblinde kontrollierte klinische Studien für Bifonazol und Terbinafin nach. Hierbei musste die klinische Diagnose sowohl durch den Nachweis von Pilzelementen im Nativpräparat als auch durch den kulturellen Pilznachweis mit Erregeridentifikation gesichert sein. Die Auswahlkriterien limitierten die Studienanzahl im Besonderen für Bifonazol. Alle Studien zu Bifonazol stammen aus den 80er Jahren, und es ist zu hinterfragen, ob sie heutigen Ansprüchen noch genügen. Für die Indikation Tinea pedis konnten lediglich zwei direkte Vergleichsstudien (Tinea pedis interdigitalis und Tinea pedis Mokassintyp) der beiden Wirkstoffe [37,38] selektiert werden.

In den plazebokontrollierten Studien ist dabei die signifikante Überlegenheit sowohl von Terbinafin als auch von Bifonazol hinreichend belegt worden. Während in den direkten Vergleichsstudien mit der Indikation Tinea pedis die Gesamtheilungsraten für Bifonazol zwischen 31 bis $63 \%$ liegen, konnten für Terbinafin Raten von 78 und $83 \%$ erzielt werden. In beiden Studien $[37,38]$ wurde eine signifikante Überlegenheit von Terbinafin nachgewiesen.

Auffallend war, dass in den Terbinafin-Gruppen sowohl die klinische als auch die mykologische Heilung deutlich früher einsetzte als unter Bifonazol. Nach 3-wöchiger Anwendung von Terbinafin konnten bereits klinische und mykologische Heilungsraten von 90\% bzw. 80\% nachgewiesen werden, während für Bifonazol lediglich Raten von $40 \%$ bzw. $25 \%$ erreicht wurden [4].

Gleiche Tendenzen zeigt die Studie von Sanchez Caruso et al. [38]. Nach 2-wöchiger Applikation von Bifonazol konnten lediglich mykologische und klinische Heilungsraten von 25\% erzielt werden, während die entsprechenden Raten für Terbinafin bereits $77 \%$ und $73 \%$ betrugen und somit deutlich höher lagen.

Der im Vergleich zu Bifonazol schnellere Wirkungseintritt unter Terbinafin wird in einer weiteren klinischen Untersuchung von Evans et al. [40] bestätigt. 65 Patienten mit Tinea pedis wurden 
für 1, 3, 5 bzw. 7 Tage einmal täglich mit $1 \%$ iger Terbinafin Creme therapiert und insgesamt drei Monate beobachtet. Eine mykologische Heilung konnte nach einmaliger Anwendung des Wirkstoffes bereits bei $94 \%$ der Patienten diagnostiziert werden. Die Gesamtheilung wies die besten Ergebnisse bei 7-tägiger Anwendung auf. Der Vergleich eines ein- bzw. 4-wöchigen Therapiezyklus zeigte keine bedeutsamen Unterschiede zwischen klinischer und mykologischer Heilungsrate, so dass bereits ein 1-wöchiger Therapiekurs zu effektiven Ergebnissen führt [41].

Fazit

Die heute weltweit therapiebestimmenden lokalen Antimykotika in der Behandlung der Tinea pedis sind Imidazole und Allylamine. Zu den in Deutschland am häufigsten angewendeten Imidazolen der neueren Generationen zählt Bifonazol. Im Vergleich zu früheren Imidazolen sind eine kürzere Therapiedauer und nur noch eine einmal tägliche Applikation erforderlich. Unter den Allylaminen ist Terbinafin der meist verwendete Wirkstoff. Auch Terbinafin muss lediglich einmal täglich appliziert werden, jedoch ist die Anwendungsdauer mit sieben Tagen bei Tinea pedis interdigitalis deutlich geringer.

Verschiedene pharmakokinetische Eigenschaften von Terbinafin rechtfertigen eine Kurzzeittherapie:

- Eine hohe intrinsische Aktivität, abgebildet durch niedrige MHK-Werte zwischen 0,001 und 0,01 mg/l gegen Dermatophyten,

- die Fähigkeit, rasch und effektiv ins Stratum corneum zu penetrieren und sich dort auch anzureichern, derart, dass eine Verfügbarkeit auch noch nach Applikationsende gesichert ist, - ein primär fungizider Wirkmodus.

Im Vergleich dazu wurden in vitro für Bifonazol bezüglich Dermatophyten höhere Hemmkonzentrationen ermittelt, mit einem fungiziden Wirkmodus gegen Dermatophyten erst ab $5 \mathrm{mg} / \mathrm{l}$. Trotz guter Penetration in die Haut ist eine Behandlungsdauer von etwa drei Wochen erforderlich.

Die überlegenen In-vitro-Eigenschaften von Terbinafin finden auch in klinischen Studien zur Wirksamkeit ihren Widerhall. Die ermittelten Heilungsraten sind zumindest gleichwertig, zeigen jedoch meist eine Überlegenheit von Terbinafin. Am deutlichsten tritt der Vorteil von Terbinafin in der Behandlungsdauer in Erscheinung. In den direkten Vergleichsstudien zwischen Bifonazol und Terbinafin konnten für Terbinafin sowohl für Tinea pedis interdigitalis als auch für Tinea pedis plantaris ein schnellerer mykologischer und klinischer Heilungseintritt und trotz kürzerer Behandlungsdauer eine geringere Rezidivrate beobachtet werden.

\section{Literatur}

${ }^{1}$ Arbeitsgemeinschaft der wissenschaftlichen medizinischen Fachgesellschaft (AWMF). Das Leitlinien-Manual von AWMF und ÄZQ. Z Ärztl Fortbild Qualitätssich 2001; 95: 1 - 84

${ }^{2}$ AWMF Deutsche Dermatologische Gesellschaft und Deutschsprachigen Mykologische Gesellschaft. Leitlinien Tinea der freien Haut, Nov. 2002, http://www.uni-duesseldorf.de/AWMF/11/derm-m01.html
${ }^{3}$ Braun-Falco O, Plewig G, Wolff HH. Dermatologie und Venerologie. Berlin: Springer Verlag, 1984: 211 - 212

${ }^{4}$ Aste N, Pau M, Aste N, Biggio P. Tinea pedis observed in Cagliari, Italy, between 1996 and 2000. Mycoses 2003; 46: 38-41

${ }^{5}$ Kemna ME, Elewski BE. A U.S. epidemiologic survey of superficial fungal diseases. J Am Acad Dermatol 1996; 35: 539- 542

${ }^{6}$ Maruyama R, Hiruma M, Yamauchi K, Teraguchi S, Yamaguchi H. An epidemiological and clinical study of untreated patients with tinea pedis within a company in Japan. Mycoses 2003; 46: 208 - 212

7 Summerbell RC, Kane J, Krajden S. Onychomycosis, tinea pedis and tinea manuum caused by non-dermatophytic filamentos fungi. Mycoses 1989; 32: 609-619

8 Stenderup A. Ecology of yeast and epidemiology of yeast infections. Acta Derm venereol (Stockh) 1986; Suppl 121: 27-37

${ }^{9}$ Berg D, Plempel M. Bifonazole, a biochemist's view. Dermatologica 1984; 169: 3-10

${ }^{10}$ Plempel M, Regel E, Büchel KH. Antimycotic efficacy of bifonazole in vitro and in vivo. Arzneimittelforschung 1983; 33: 517-524

${ }^{11}$ Yamaguchi H, Hiratani T, Plempel M. In vitro studies of a new imidazole antimycotic, bifonazole, in comparison with clotrimazole and miconazole. Arzneim-Forsch Drug Res 1983; 33: 546 - 555

${ }^{12}$ Seebacher C. Dermatomykosen - Grundlagen und Therapie. Heidelberg: Springer Verlag, 2001: 41

${ }^{13}$ Patzschke K, Ritter W, Siefert HM, Weber H, Wegner LA. Pharmacokinetic studies following systemic and topical administration of $\left[{ }^{14} \mathrm{C}\right] \mathrm{bi}-$ fonazole. Arzneim-Forsch 1983; 33: 745-750

14 Plempel M, Berg D, Ritter W. Bifonazole, a new topical azole antimycotic with specific properties. In: Hay RJ. Advances in topical antifungal therapy. Berlin: Springer Verlag, 1986: 4-20

${ }^{15}$ Ryder NS. The mechanism of action of terbinafine. Clin Exp Dermatol 1989; $14: 98-100$

${ }^{16}$ Ryder NS. Terbinafin: mode of action and properties of the squalene epoxidase inhibition. Br J Dermatol 1992; 126: 2-7

${ }^{17}$ Faergemann J, Zehender H, Jones T, Maibach I. Terbinafine levels in serum, stratum corneum, dermis-epidermis (without stratum corneum), hair, sebum and eccrine sweat. Acta Derm Venereol 1991; 71: $322-326$

${ }^{18}$ Hill S, Thomas R, Smith SG, Finlay AY. An investigation of the pharmacokinetics of topical terbinafine $\left(\right.$ Lamisil $^{\circledR}$ ) $1 \%$ cream. Br J Dermatol 1992; 127: $396-400$

19 Tanuma H, Doi M, Ohta Y, Nishiyama S, Katsuoka K, Kaneko S, Mukai H, Abe M. Usefulness of $1 \%$ terbinafine $\mathrm{HCl}\left(\right.$ Lamisi $\left.^{\circledR}\right)$ cream for hyperkeratotic-type tinea pedis and its transfer into the horny layer. Mycoses 2000; 43: 417-432

${ }^{20}$ Dykes PJ, Thomas R, Lever I, Marks R. Pharmacokinetics of topically applied terbinafine: results from studies in healthy volunteer subjects and patients with pityriasis versicolor. J Dermatol Treat 1990; 1: $19-$ 21

${ }^{21}$ Clayton YM. In vitro activity of terbinafine. Clin Exp Dermatol 1989; 14: $101-103$

${ }^{22}$ Korting HC, Ollert M, Abeck D. Results of German multicentre study of antimicrobial susceptibilities of Trichophyton rubrum and Trichophyton mentagrophytes strains causing tinea unguium. Antimicrob Agents Chemother 1995; 39: $1206-1208$

${ }^{23}$ Petranyi G, Meingassner JG, Mieth H. Antifungal activity of the allylamine derivative terbinafine in vitro. Antimicrob Agents Chemother 1987; 31: $1365-1368$

${ }^{24}$ Carrillo-Munzo AJ, Tur-Tur C. Comparative study of antifungal activity of sertaconazole, terbinafine, and bifonazole against clinical isolates of candida spp., Cryptococcus neoformans and dermatophytes. Chemotherapy 1997; 43: $387-392$

25 Bagatell FK. Elimination of dermatophytes causing tinea pedis interdigitalis with once-daily application of bifonazole $1 \%$ solution. Adv Ther 1986; 3: $265-271$

${ }^{26}$ Coffey WJ. Management of tinea pedis interdigitalis with bifonazole $1 \%$ cream - double-blind study. Adv Ther 1986; 3: 301 - 307

${ }^{27}$ Izuno GT. A double-blind study of bifonazole $1 \%$ cream applied once daily in the treatment of tinea (pityriasis) versicolor and tinea pedis interdigitalis. Adv Ther 1986; 3: 308 - 316

${ }^{28}$ Smith EB, Tschen E. Treatment of interdigital tinea pedis and tinea (pityriasis) versicolor with bifonazole $1 \%$ solution applied once daily. Adv Ther 1986; 3: 250-256

${ }^{29}$ Berman B, Ellis C, Leyden J, Lowe N, Savin RC, Shupack J, Stiller M, Tschen E, Zaias N, Birnbaum JE. Efficacy of a 1 week, twice-daily regimen of terbinafine $1 \%$ cream in the treatment of interdigital tinea 
pedis: results of placebo-controlled, double-blind, multicentre trials. J Am Acad Dermatol 1992; 26: 956-960

${ }^{30}$ Hollmen KA, Kinnunen T, Kiistala U, Väänänen A, Saarelainen IO, de Cuyper C, Decroix J, Broeckx W, Karvonen J. Efficacy and tolerability of terbinafine $1 \%$ emulsion gel in patients with tinea pedis. Eur Acad Dermatol Venerol 2002; 16: 87-88

${ }^{31}$ Korting HC, Tietz HJ, Bräutigam M, Mayser P, Rapatz G, Paul C. One week terbinafine $1 \%$ cream (Lamisil) once daily is effective in the treatment of interdigital tinea pedis: a vehicle controlled study. Med Mycol 2001; 39: 335 - 340

${ }^{32}$ Lebwohl M, Elewski B, Eisen D, Savin RC. Efficacy and safety of terbinafine $1 \%$ solution in the treatment of interdigital tinea pedis and tinea corporis or tinea cruris. Cutis 2001; 67: 261 - 266

${ }^{33}$ Savin RC. Treatment of chronic tinea pedis (athlete's foot type) with topical terbinafine. J Am Acad Dermatol 1990; 23: 786 - 789

${ }^{34}$ Smith EB, Noppakun N, Newton RC. A clinical trial of topical terbinafine (a new allylamine antifungal) in the treatment of tinea pedis. J Am Acad Dermatol 1990; 23: 790-794

${ }^{35}$ Savin RC, Atton AV, Bergstresser PR, Elewski B, Jones HE, Levine N, Leyden J, Monroe A, Pandya A, Shupack J, Stiller M, Tschen E, Weinstein G, Zaias N, Birnbaum JE. Efficacy of terbinafine 1\% cream in the treatment of moccasin-type tinea pedis: Results of placebo-controlled multicentre trials. J Am Acad Dermatol 1994; 30: 663-667

${ }^{36}$ Evans EGV, James IGV, Joshipura RC. Two-week treatment of tinea pedis with terbinafine (Lamisil) $1 \%$ cream: a placebo controlled study. J Dermatol Treatm 1991; 2: 95-97

37 Wahid Z, Butt J, Nishtar R. Multicentre double-blind study of efficacy and safety of terbinafine $1 \%$ cream compared with bifonazole $1 \%$ cream in patients with tinea pedis moccasin type. J Dermatol Treat 1997; 8: $225-227$

${ }^{38}$ Sanchez Carazo JL, Fuente C, Oliver V, Umbert P. Estudio comparativo de terbinafina vs. bifonazol crema al $1 \%$ en aplicacion unica diaria en tina pedis. Actas-Dermo Sif 1994; 85: $388-394$

${ }^{39}$ Galimberti RL, Belli L, Negroni R, Castr JM, Rohwedder R, Tuculet MA. Prophylaxis of tinea pedis interdigitalis with bifonazole, $1 \%$ powder. Dermatologica 1984; 169: 111 - 116

40 Evans EGV, Seaman RAJ, James IGV. Short-duration therapy with terbinafine $1 \%$ cream in dermatophyte skin infections. Br J Dermatol 1994; 130: $83-87$

${ }^{41}$ Sugiura M, Hata Y, Fukuda T, Ishizaki S, Hanyaku H, Naka W, Harada T, Nishikawa T. One-week application of terbinafine cream compared with four-week application in treatment of tinea pedis. Nippon Ishinkin Gakkai Zasshi 2001; 42: 223-228

\section{Beauty Care Preis der Arbeitsgemeinschaft für ästhetische Dermatologie und Kosmetologie (ADK) e.V.}

Die ADK schreibt 2004 zum dritten Mal den mit 5000 Euro dotierten Beauty Care Preis aus für herausragende und wegweisende Forschungsarbeiten auf dem Gebiet der ästhetischen und kosmetischen Dermatologie. Arbeiten aus der Grundlagenforschung und Klinischen Forschung können in deutscher und englischer Sprache bis zum 1.11.2004 in 2-facher Fertigung (Postweg) eingereicht werden an:

Prof. Dr. med C. Bayerl

Universitätsklinik für Dermatologie,

Venerologie und Allergologie Mannheim

Theodor-Kutzer-Ufer 1 - 3

68135 Mannheim

Tel. 0621/383-3918

Fax: 0621/383-3815

E-mail: christiane.bayerl@haut-ma.uni-heidelberg.de

Zugelassen sind wissenschaftliche Publikationen in anerkannten Fachzeitschriften, die innerhalb der letzten 2 Jahre publiziert oder zur Veröffentlichung angenommen wurden, zum Thema: Ästhetische Dermatologie und Kosmetologie.

Der Beitrag darf bei keiner anderen Ausschreibung eingereicht werden oder eingereicht worden sein. Jeder Autor kann nur eine Arbeit einreichen. Die Zustimmung von Mitautoren muss in schriftlicher Form beigefügt werden. Das Recht der Publikation bleibt beim Autor. Zur Teilnahme berechtigt sind in Deutschland, Österreich oder der Schweiz tätige Dermatologen aus Klinik und Praxis.

Über die Verleihung des Preises entscheidet eine fünfköpfige Jury, bestehend aus universitären und niedergelassenen Fachkollegen. Bei Vorliegen qualitativ gleichwertiger Arbeiten kann der Preis auch geteilt werden, zwischen einer Arbeit aus der Grundlagenforschung und einer Arbeit aus der Klinischen Forschung. Der Preisträger und die übrigen Bewerber werden über die Entscheidung der Jury vor Preisverleihung schriftlich informiert.

Der Preis wird verliehen bei der 10. Münchner Fortbildung für Dermatologie und Kosmetik (MFFDK) am 4./5.12. 2004 in München.

Mögliche Themen sind:

- Aufklärung der Wirkprinzipien kosmetischer Behandlungen und Präparate

- Neue Methoden zur Bewertung der Wirkung

- Entwicklung innovativer Anwendungsformen oder Technologien

- Neue Methoden zur Nutzen/Risiko- Abschätzung kosmetischer Mittel und Maßnahmen

- Allergie und Kosmetik

- UV und Kosmetik

- Hautverjüngung/Hautalterung 